\title{
Duration of Collaboration from a Market Perspective: An Agent-based Modeling Approach
}

\author{
Niniet I. Arvitrida \\ Department of Industrial Engineering, \\ Sepuluh Nopember Institute of Technology, Surabaya 60111 Indonesia \\ Email: niniet@ie.its.ac.id (Corresponding Author) \\ Antuela A. Tako \\ School of Business and Economics, \\ Loughborough University, Loughborough, LE11 3TU UK \\ Email: A.Takou@lboro.ac.uk \\ Duncan A. Robertson \\ School of Business and Economics, \\ Loughborough University, Loughborough, LE11 3TU UK \\ Email: D.A.Robertson@1boro.ac.uk \\ Stewart Robinson \\ School of Business and Economics, \\ Loughborough University, Loughborough, LE11 3TU UK \\ Email: S.L.Robinson@lboro.ac.uk
}

\begin{abstract}
Maintaining a long-term partnership with a supplier is considered an effective strategy to achieve collaboration success in supply chain management (SCM). However, individual companies find that this approach does not always improve business performance. In this paper, an agent-based model (ABM) is developed to investigate the effect of duration of collaboration on supply chains from a market perspective. The model represents two-stage supply chains of an innovative product market, involving suppliers and manufacturers. The model outputs are measured by the rate of demand fulfilment and the number of supply chains which can survive in the market. The results show that duration of collaboration has no significant impact on both demand fulfilment and survivability of supply chains. This finding contradicts the common belief held in the literature about the benefits of longterm collaboration, but it corroborates examples encountered in practice. This study provides new insights to the practice of supply chain collaboration by taking a market perspective. The results show that a longer duration of collaboration does not provide a significant improvement to the supply chain's competitiveness from a market point of view, in terms of demand fulfilment and supply chain's ability to survive over the long-term. The implications of this finding to practice are discussed in the paper.
\end{abstract}

Keywords: supply chain, duration of collaboration, market perspective, agent-based modeling, simulation

\section{INTRODUCTION}

In most supply chain management (SCM) literature, long-term collaboration strategy has been generally understood as an effective approach to optimize a supply chain's competitive advantage. It promotes better communication between collaborating firms and accelerates the innovation process along the supply chain (Boddy et al. 1998). The strategy can also secure the supply flow, particularly when it is applied along with single-sourcing strategy, where the manufacturer only collaborates with one supplier (Kraljic 1983). Furthermore, this strategy can reduce the time to market of a new product as it speeds up the product innovation process through supplier involvement (Christopher 2000). These claims of collaboration advantages are also supported by the successful SCM practices in several large companies, such as Toyota and Benetton, Dow Chemical, BASF, General Motors, and DuPont.

On the other hand, from a market point of view, a long-term collaboration does not always lead every supply chain to success. Many firms failed to achieve successful collaborations even though they have adopted this strategy. When this collaboration strategy does not provide the impact as expected, the operations of the supply chain turns to be less effective and inefficient (Barratt, 2004; Cao and Zhang, 2011; Holweg et al. 2005; Lambert and Cooper, 2000). Several studies also suggest that this collaboration strategy does not always enhance supply chain 
performance, investigated by Anderson and Jap (2005), Burke et al. (2007), Leeuw and Fransoo (2009), Squire et al. (2009), and Sun and Debo (2014). In addition, from a strategic management's perspective, as recommended by Porter (1997), long-term close partnerships may hinder firms to innovate and improve their performance as the strategy can deter the benefit of competition.

The contradicting views above serve as the motivation for the paper, which aims to understand the effect of duration of collaboration on supply chains. Furthermore, the literature points out that longer term collaboration does not always improve supply chain competitiveness. Hence, this study aims to gain a better understanding of the effect of duration of collaboration on supply chains, by analyzing the supply chains from a market perspective.

An agent-based modeling (ABM) approach is adopted in order to incorporate the dynamics of partnerships in supply chains that are driven by individual firm behavior. The approach also allows system level observation on the emergent impact of the individual firm behavior from a market perspective. This approach offers a novel perspective in supply chain analysis, particularly in exploring the impact of a supply chain collaboration strategy over the long-term of competition.

In this paper, we assess the impact of duration of collaboration on the supply chains competitiveness over the long term measures against the supply chains ability to meet the customer demand (demand fulfillment) and the number of supply chains that survive in the market. The hypothesis tested in this study is "longer duration of collaboration does not increase supply chain's competitiveness, in terms of ability to fulfil the demand and survive over the long term in the market". This hypothesis is formalized based on the contradicting views on the effect of long-term collaboration found in the literature.

The remaining sections of this paper are organized as follows. First, a brief review of the duration of collaboration SCM literature is presented followed by an overview of agent-based modeling. Then, the agent-based model is explained in section 4. The experiments and results are discussed in section 5, followed by the discussion of the findings.

\section{DURATION OF}

\section{COLLABORATION IN SUPPLY CHAINS}

Collaboration with suppliers is one SCM strategy to improve supply chain performance. This strategy allows suppliers to be involved earlier in the process of product development, so both supplier and manufacturer can obtain long-term joint competitiveness, as practiced by Benetton with its supplier (Dapiran 1992). Moreover, it promotes a better efficiency and lower output variability since this method reduces the number of suppliers that affect transaction costs (Matthyssens and Van den Bulte 1994).

The most effective collaboration suggested are the ones where the manufacturers in the supply chain establish a long-term relationship. This strategy has been widely accepted as a basic approach in SCM to improve and optimize supply chain competitiveness over the long term (Matthyssens and Van den Bulte 1994; Li et al. 2006). The strategy can secure the supply of critical materials or components of the finished products (Kraljic 1983). Kraljic also documented that a Japanese steel industry has decreased their total spending by up to $18 \%$ by applying this partnership style. In addition, long-term collaboration enables information and operations integration that improves supply chain performance (Prajogo and Olhager 2012). Using a game theory model, Ren et al. (2010) also support the benefit of long-term collaboration that can improve the quality of information sharing for sales forecast and facilitate trust between collaborating enterprises.

However, intense partnerships through long-term collaboration do not always have positive impacts on the firms. Failures on partnerships have been found at a relatively high rate, which is between $30 \%$ and $50 \%$ (Anderson and Jap, 2005). In addition, Parker and Hartley (1997) find that long-term partnerships tend to make suppliers to be vulnerable to losing their power to control the price of the material. Kraljic (1983) also suggests that long-term partnerships would provide a significant benefit if the suppliers operate beyond their capacity, and the supply market is highly uncertain. Furthermore, Porter (1997) does not recommend long-term collaboration as it can reduce supplier's willingness to innovate.

In addition, several studies suggest that long-term relationships lead the partners to be more likely dissatisfied with the cooperative arrangement. Several studies in marketing management found empirically that long term relationships can lower trust and the service performance (Grayson and Ambler 1999). This finding is supported by studies in strategic management which posits that close partnerships can encourage the collaborating partner to be too dependent on each other (Inkpen and Beamish 1997). In addition, when a firm has a better understanding of what the other knows through a high degree of information sharing between parties, the partnerships become unstable and fragile. This risk will be more apparent if the demand uncertainty is very high or very low (Sun and Debo 2014). In the case of Japanese car manufacturers, Dyer and Ouchi (1993) find that a long-term collaboration does not necessarily need a very high involvement of collaborating firms. In addition, the long-term relationships strategy is also often doubted by SCM practitioners. This strategy is viewed to be risky as developing and maintaining trust between firms are difficult in business relationships. This negative opinions often come from suppliers with larger scale buyers who aggressively established strategic and long-term cooperation with them (Bensaou 1999).

An empirical study conducted by Wagner (2011) suggests that the length of partnership has no relationship with performance improvement. Instead, according to Wagner (2011), supply chain performance is influenced by the level of supplier development - a set efforts of a manufacturer spends to enhance supplier performance and/or capabilities. However, the effectiveness of supplier development tends to follow a curve-linear pattern against the length of partnership. It implies that excessive duration of relationship would not provide a significant benefit to the success of supplier development.

This suggestion is consistent with the study conducted by Squire et al. (2009) who also conclude that collaboration 
has a curve linear relationship. It improves manufacturer's responsiveness, but when manufacturer becomes dependent on the supplier, it may have an adverse impact on suppliers' performance. However, both Wagner (2011) and Squire et al. (2009) do not explain the length of relationships duration for a specific unit of time.

In summary, contradicting views on the effect of longterm partnerships on supply chains is found in the literature. This gap indicates that longer duration of collaboration does not always benefit every single supply chain in the market. Hence, this study investigates this issue by considering all competing supply chains from a market perspective. The next section provides an overview of the modeling approach employed in this paper as well as framing the existing literature of ABM in supply chain competition and collaboration.

\section{OVERVIEW OF AGENT-BASED MODELING}

Investigating the effect of duration of collaboration from a market perspective is hard to perform empirically. It will consume a great amount of cost and time, as well as causing potential problems related to ethics as it requires high level of transparency in formalizing firm's behavior in strategic decisions. Therefore, agent-based modeling $(\mathrm{ABM})$ is an appropriate approach for studying supply chain collaboration with market-level viewpoint.

In supply chain operations, $\mathrm{ABM}$ has been a growing body of research with many applications, such as manufacturing, telecommunications, transportation systems, information management, interactive entertainments, and healthcare (Jennings et al. 1998). The agents are commonly described as companies with decision-making intelligence to manage sourcing, stocking, and shipping (Macal and North 2011). However, compared to discrete-event simulation (DES) and system dynamics (SD), the use of ABM in supply chain analysis is still limited to date. No paper has reviewed the applications of ABM in SCM, while the use of DES and SD has been discussed in depth by Tako and Robinson (2012). Where $\mathrm{ABM}$ has been applied to the SCM context, it is mostly conducted through computer science research. The work tends to focus on software architecture rather than analyzing collaboration from a market approach. Moreover, $\mathrm{ABM}$ is still a relatively new tool to operational research, compared to DES and SD (Onggo and Karatas 2015). The following subsections briefly discuss the features of $\mathrm{ABM}$ and the previous ABM research in a SCM context.

\subsection{Features of $A B M$}

ABM consists of five main features: the agent, the environment, interactions, autonomy, and the schedule. An ABM simulation model is composed of a set of agents acting in an environment. The agent represents the intelligence object that we want to simulate. It can make a decision without explicit guidance of humans or other agents (Wooldridge and Jennings 1995). The agents are modelled individually to create the system. They take actions autonomously based on particular interaction rules, and these individual actions generate the global patterns of the system. The agents can be sociable and interdependent, so they can influence the others in response to these observations. Meanwhile, the environment is the abstract space where the agents are populated. It can be a twodimensional world in the simplest representation (Robertson and Caldart 2009) or N-dimensional space (Robertson 2003). Agent's positions in the environment influences their state towards other agents and their decision during their interaction with the others. Lastly, the rules of agent refer to the detail interaction, the autonomy of behavior of an individual agent. This feature leads the individual actions or decisions of agents. A simple rule of the agent can lead to complexity represented as the emergent pattern in the system. The actions are executed based on timescales, or schedules, which is prosecuted either discretely as in discrete event or concurrently. The schedule controls whether all agents act at the same time or in a particular order (Collier 2003; Robertson and Caldart 2009).

\subsection{Existing ABM work in SCM}

Several SCM issues have been examined by using an ABM approach. Several studies regard supply chain collaboration, such as Xue et al. (2005), Zhang et al. (2006), and Zhu (2008). Xue et al. (2005) employ ABM to address the collaboration issue in construction supply chain, but they concentrate on the information flow and negotiation. Zhang et al. (2006) present an ABM as an approach for e-manufacturing to provide flexibility, robustness, and adaptability to the rapid changes. Zhu (2008) also models supply chain collaboration, but it does not consider the collaboration as integration between firms; the study focuses on the impact of information sharing in a single two-stage supply chain. Other studies consider supply chain collaboration only in the scope of inventory decisions, such as Dimitriou (2010) who examines the effect of boundedly rational decisions in the Newsvendor inventory model. Nevertheless, this research is limited to a single supply chain.

In addition, ABM studies in SCM concentrate mostly on software architecture rather than investigating the problem. The research tends to employ $\mathrm{ABM}$ as a part of intelligent system in decision making rather than solely using it for simulation. The following are several examples of these studies described in brief. Swaminathan et al. (1998) utilize ABM as a multi-agent approach to develop a supply chain modeling framework, then addresses supply chain configuration, coordination, and contract issues, which deal with inventory decisions. Julka et al. (2002) propose an ABM framework for developing a decision support system prototype to integrate supply chain processes in a refinery supply chain. However, the goal of the system is optimizing a firm's performance, not the supply chain. Jiao et al. (2006) apply an ABM system to develop a framework of collaborative negotiation in a supply chain. The framework incorporates supply chain network and inventory decisions. Cheng (2011) proposes an agent-based supply chain collaboration model that studies production and logistics processes at enterprise-level. The model comprises a single two-stage supply chain, which involves a manufacturer and a supplier. It considers competition to the model, but the competition is only represented by achieving on-time delivery target. 
Based on above review, little mention is made of the use of ABM for supply chain analysis. All of these studies observe a particular single supply chain and none of them view supply chain problems from the market perspective. In short, research that analyses firms' behavior in competition and collaboration by using an ABM approach has not been carried out yet in SCM. The following section presents an example of ABM application to analyze SCM problem from market perspective. An issue of competition and collaboration is addressed, by focusing on the duration of collaboration in particular.

\section{AGENT-BASED MODEL OF COMPETITION AND COLLABORATION IN SUPPLY CHAINS}

This study considers supply chains of an innovative product market, where partnerships between manufacturer and supplier are critical to the supply chain competitiveness. Having this scope, the agents are defined as the actors along the supply chains, which involve customers, manufacturers, and suppliers.

The customer is considered as a group of customers, retailers, or a warehouse. Each customer has a fixed preference in buying the product, represented by a fixed position of customer in the simulation space (they do not move). The customer also has a compromise limit to reflect the maximum degree of customer's willingness to compromise toward their product preference. This compromise reflects a feature of innovative product market where not all customers buy the product. Each customer selects the closest manufacturer which is close to its buying preference and stays within the limit of the customer's willingness to compromise.

As the customer, the manufacturer also has a preference in selecting a supplier and willingness to compromise. The preference is reflected by the manufacturer's position in the simulation space, and the willingness to compromise represents the tolerable capability gap between the manufacturer and the supplier when the manufacturer has to link with less efficient and/or responsive supplier/s. The willingness to compromise also represents the degree of supplier/s' impact on the manufacturers or supply chain capability during competition and collaboration. This effect can limit the manufacturers' competition movement as the manufacturers' competitiveness is affected by the suppliers. The suppliers compete to attract manufacturers to cooperate with them, and manufacturers continuously move to obtain more customers. They have no trust/loyalty to each other, and they can die when they cannot manage to have a collaboration link within several periods.

The agents act in a two-dimensional environment, which represents their strategic position from an SCM perspective. The dimensions are referred to supply chain competitive strategy as described by Chopra and Meindl (2007), namely efficiency and responsiveness. Within the environment, two infeasible areas reflect the limits to the possible competitive landscape, which is presented in Figure 1. The infeasible area which is on the left defines unrealistic extreme firm competitiveness by offering a product with both a high level of innovation or customization and a very low price and product value to the customers. The opposite extreme competitiveness is described by the other infeasible area: a firm is impossible to sell non-innovative products with an extremely expensive price and high perceived product value.

On the other hand, the feasible area reflects a simplified non-linear relationship between responsiveness and efficiency in supply chain practice. A higher responsiveness level of supply chain operations can either enhance or decrease the supply chain efficiency. In contrast, if a supply chain is highly efficient, the supply chain will be very likely less responsive. In short, higher responsiveness levels can lead to much wider range of efficiency degree (zone $a$ in Figure 1) compared to higher efficiency levels that restrict the resulting responsiveness level (zone $b$ in Figure 1). In the model, this relationship affects the shape of the feasible area, which the length of zone $a$ is assumed to be 3 times longer or wider than zone $b$.

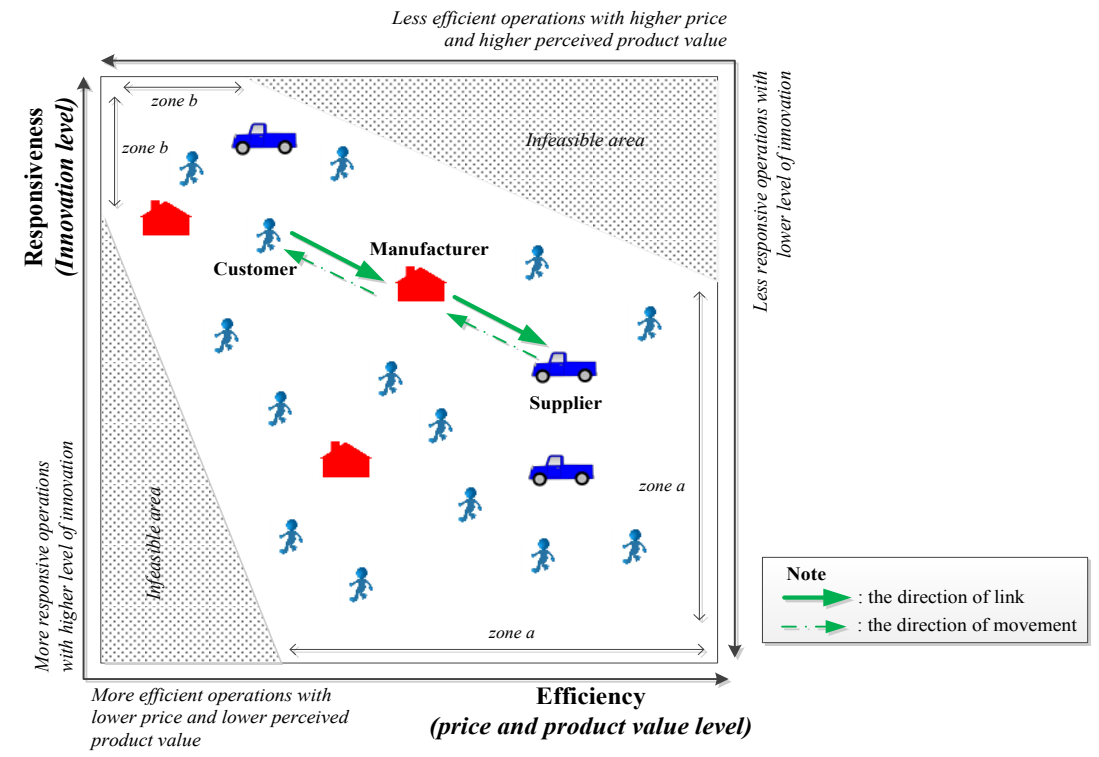

Figure 1 An abstraction of the agents within their environment 
The time unit of the simulation run represents a period that is considered sensible to allow a firm to make a slight adjustment to their strategic position. Regarding the operations in supply chains, the duration of making a slight strategic change can be related to the time bucket used in the sales and operations plan (SOP). The time bucket commonly used in the SOP is between 3 and 18 months, which are mostly affected by the total production lead time. In the computer model, we assume that one time unit represents at least 3 months for making a gradual strategic change. The definition of time unit controls all actions of the agents, which defines the schedule of agent-based model.

The main outputs or responses of this model are the demand fulfilment rate and the number of supply chains in the market. The demand fulfilment rate represents the aggregate performance of the supply chains in the market, measured by the proportion of demand fulfilled with respect to the total available demand. For instance, if the available demand in the market is 100 customers and the available supply chains serve only 10 customers, the demand fulfilment rate is equal to $(10 / 100) \times 100 \%=10 \%$. Meanwhile, the number of supply chains in the market indicates the number of supply chains which can survive in long term competition.

To simplify the detail of the model, a conceptual model (Table 1) is utilized to describe the modeling objectives, inputs or experimental factors, outputs or responses, content, assumptions and simplifications of the model. The structure is adapted from Robinson (2008), and the ABM features incorporated to the structure are based on Macal and North (2013) and Robertson (2003). The flowchart of the behavioral rules is presented in Figure 2. The agents are scattered randomly on the feasible area to depict their positions corresponding to the others.

Table 1 The conceptual model

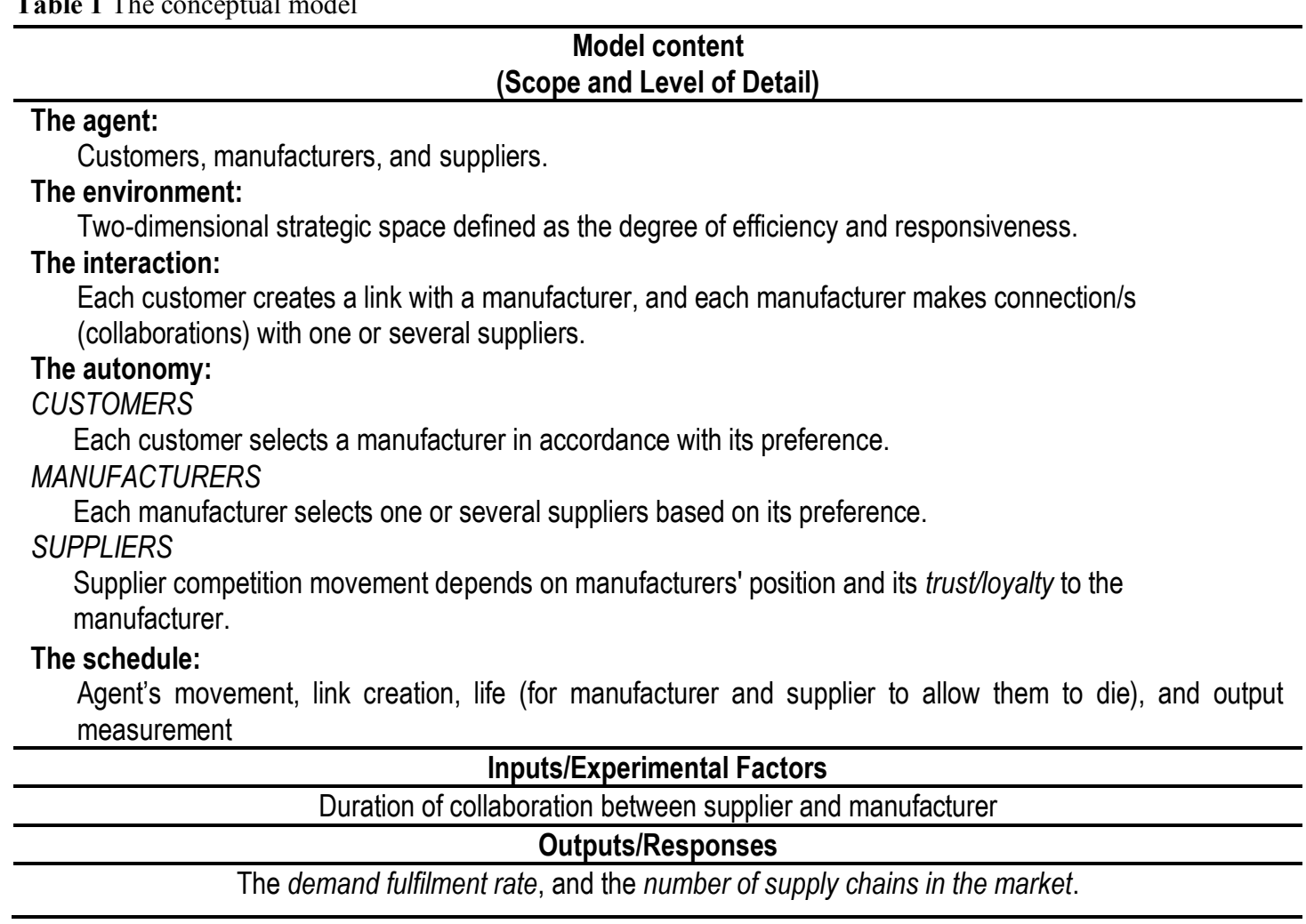




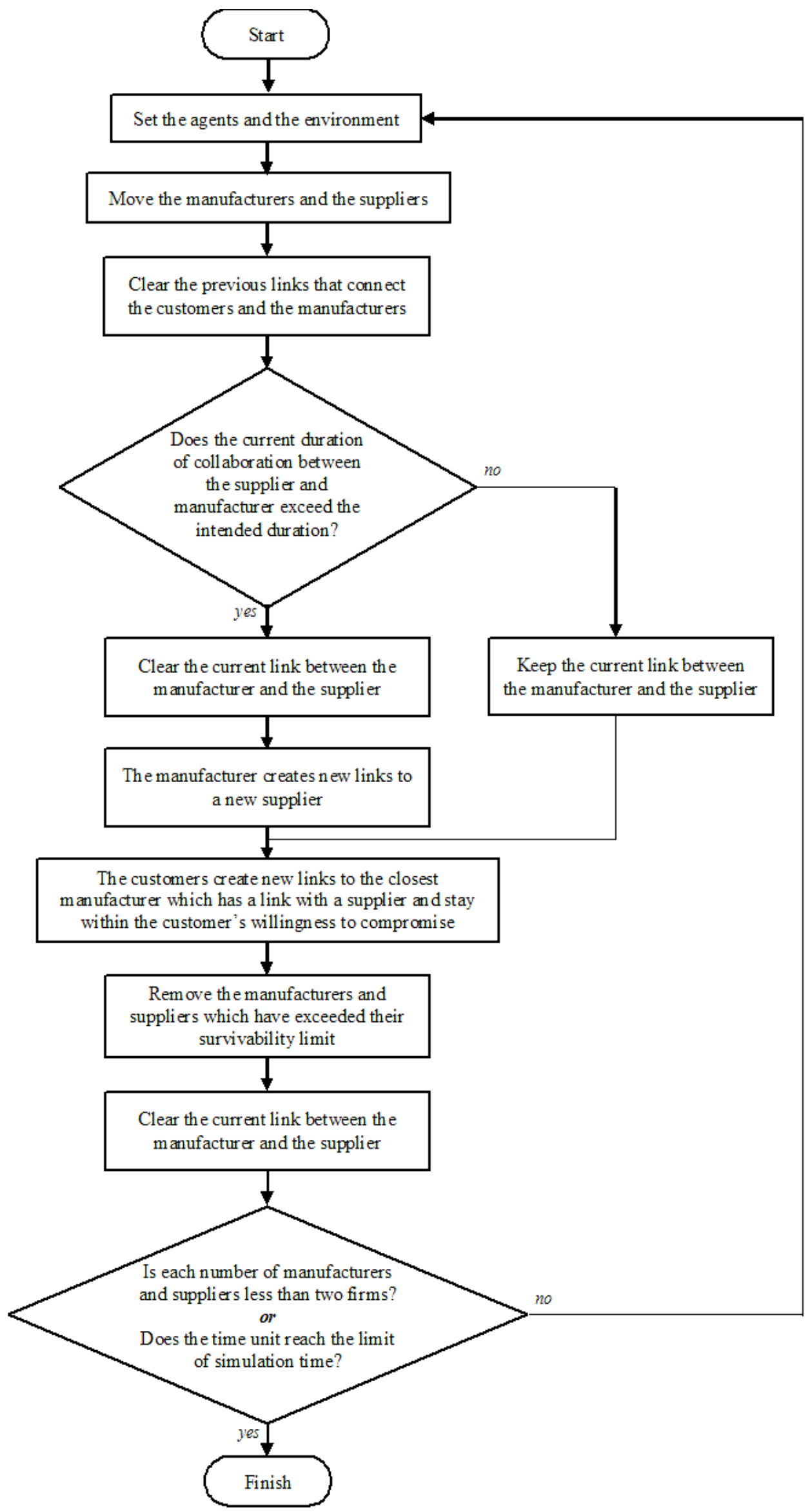

Figure 2 The agent's rules 


\section{EXPERIMENTS AND RESULTS}

The duration of collaboration is observed in five scenarios: extremely short duration, short-medium duration, medium-long duration, long duration, and extremely long duration. In the computer model, these duration levels are represented in 4 time units, 20 time units, 40 time units, 60 time units, and 80 time units. If the time unit is interpreted in the shortest possible period, which is 3 months, each scenario can be explicated as 1 year, 5 years, 10 years, 15 years, and 20 years. In consecutive, each scenario represents extremely short duration of collaboration for scenario 1, short-medium duration of collaboration for scenario 2, medium-long duration of collaboration for scenario 3, long duration of collaboration for scenario 4 , and extremely long duration of collaboration for scenario 5 .

These experimental levels are justified hypothetically since the definition of the length of collaboration depends on the detail characteristics of the innovative products, particularly in relation to supply and demand markets. For instance, ten-year collaboration for a supply chain (40 time units, in scenario 3) can be considered as a medium duration, while other supply chain may regard the duration as a long-term partnership. A summary of the scenarios is presented in Table 2. These scenarios are simulated under two different situations: one-to-one partnerships and one-tomany partnerships.

Table 2 The scenarios of duration of collaboration

\begin{tabular}{ccc}
\hline & $\begin{array}{c}\text { Duration of } \\
\text { collaboration }\end{array}$ & Scale representation \\
\hline Scenario 1 & 4 time units & Extremely short duration \\
Scenario 2 & 20 time units & Short-medium duration \\
Scenario 3 & 40 time units & Medium-long duration \\
Scenario 4 & 60 time units & Long duration \\
Scenario 5 & 80 time units & Extremely long duration \\
\hline
\end{tabular}

Firstly, the duration of collaboration is investigated in one-to-one partnerships, where both manufacturers and suppliers only allow having one collaboration link. A prominent feature is a longer duration of collaboration that does not improve the demand fulfilment rate when the suppliers are linked with only one manufacturer. Only the medium-long-term collaboration (40 time units, scenario 3) has a slightly better demand fulfilment rate than other scenarios, but it does not seem significant compared to the other scenarios. Similar interpretation is also obtained for the other model output, which is the number of supply chains which can survive in the market. The boxplots of these outputs is presented in Figure 3.

To assess whether there is a significant difference among these scenarios, the Mann-Whitney $U$ test is performed. This method is chosen as the samples are from different populations. Moreover, the data of simulation outputs is not normal, and removing the outliers will potentially lead to a less robust analysis. The one-tailed approach is used to infer which scenario has a better performance. The alternative hypothesis of this test is that the scenario with a higher median (which has a smaller Uvalue) is significantly different from the other.

Ten pairwise comparisons with normal approximation are constructed, and Bonferroni correction is applied
(Robinson 2014) with $10 \%$ overall level of significance (the critical value is -2.33 with normal approximation). Based on the comparisons, it is suggested that the demand fulfilment rate resulted in scenario 3 is significantly higher than scenario 5 , but this output is not different significantly when it is compared to the remaining scenarios. Meanwhile, the results of other pairwise comparisons to other scenarios concludes that there is no significant difference between the scenarios. It means that, overall, the longer duration of collaboration does not have a significant effect on the demand fulfilment for the long term. A similar conclusion is resulted for the number of supply chains in the market. The longer duration of collaboration does not improve the number of supply chains in the market.

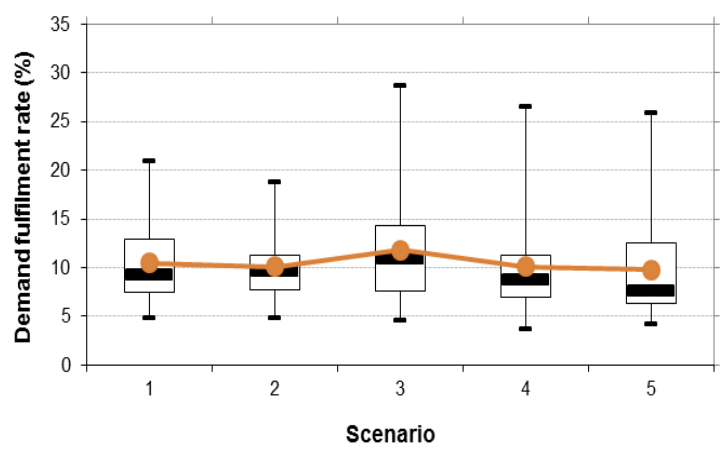

(a)

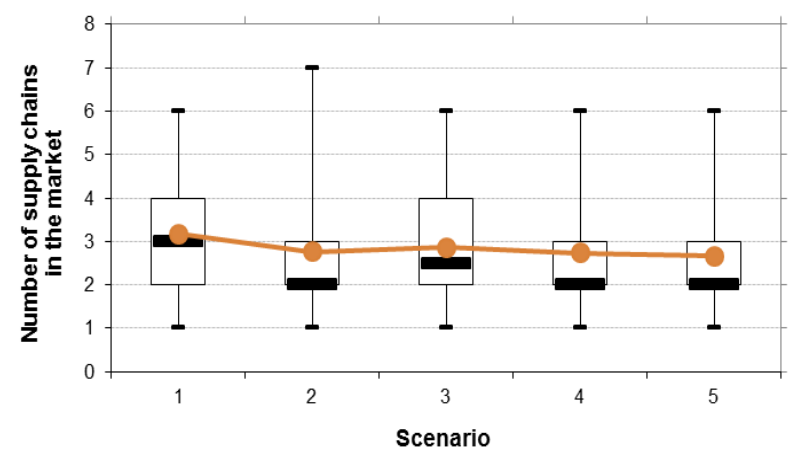

(b)

Figure 3 Boxplots of the model outputs with a line of mean values in situations of one-to-one partnerships:

a) the demand fulfilment rate, b) the number of supply chains in the market

This finding is slightly different when the scenarios are run under one-to-many partnerships, which the maximum number of supplier's partnerships is two manufacturers and manufacturer's number of partnerships remains constant at one supplier. As shown by Figure 4, unexpectedly, the extremely short duration (scenario 1) provides the highest demand fulfilment rate, with $9.68 \%$ and $9.05 \%$ for the mean and the median respectively. Meanwhile, the extremely long duration results in the lowest demand fulfilment rate, with $7.73 \%$ for the mean and $7.15 \%$ for the median. 


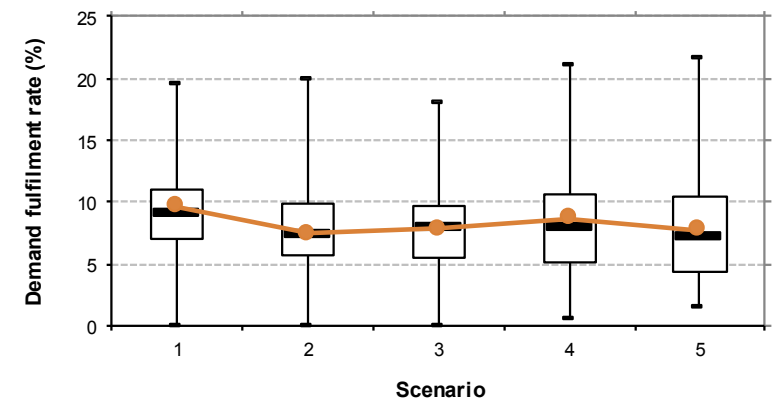

(a)

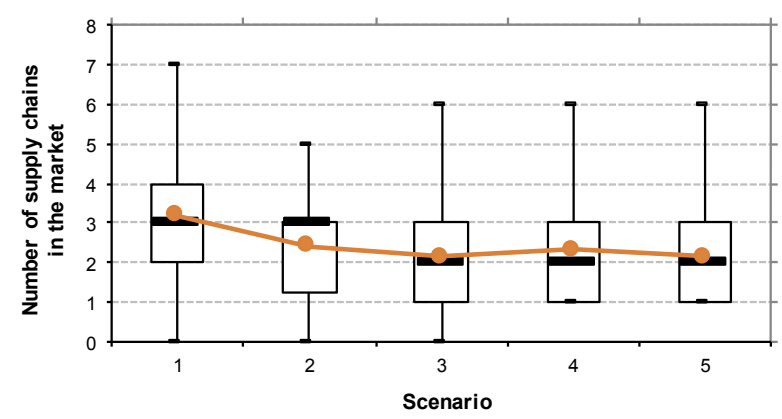

(b)

Figure 4 Boxplots of the model outputs with a line of mean values in situations of one-to-many partnerships:

a) the demand fulfilment rate, b) the number of supply chains in the market

The Mann-Whitney U test confirms that only scenario 1 has a significantly higher demand fulfilment rate than scenario 2 and scenario 5 . The difference is insignificant when it is compared to scenario 3 and 4. Meanwhile, scenario 2, scenario 3 , scenario 4 and scenario 5 are found not significantly different, in terms of demand fulfilment rate. In addition, considering the number of supply chains in the market, the results of Mann-Whitney U test show that scenario 1 provides the most significant difference compared to other scenarios, but it is not significantly different compared to scenario 2. Scenario 1 has a higher number of supply chains in the market compared to scenario 3 , scenario 4, and scenario 5. Further discussion of these results is presented in the next section.

\section{DISCUSSION OF THE FINDINGS}

This paper presents an agent-based model of supply chain collaboration to explore the effect of length of collaboration from a market perspective. The model focuses on partnerships between suppliers and manufacturers which are prominent in innovative product markets.

In the model, it is expected that a longer duration of collaboration can improve the ability to survive or the existence of both the manufacturer and the supplier in the market. However, the results of this paper indicate that when both the manufacturers and the suppliers can collaborate with one firm only at the same time (i.e. under one-to-one partnerships), this factor does not provide significant improvement to both demand fulfilment and survivability of the supply chains. On the other hand, when the supplier can work with up to two manufacturers while the manufacturers can only collaborate with one supplier (i.e. under one-tomany partnerships), the extremely short duration of collaboration (scenario 1) seems to provide better demand fulfilment and survivability of the supply chains for a longterm compared to scenarios of longer duration of collaboration (scenario 2, scenario 3, scenario 4, and scenario 5). This indicates that the adoption of long-term duration of collaboration does not lead to a better demand fulfilment and survivability levels of the supply chains in the market.

This finding contradicts the commonly held belief in SCM literature which supports the benefit of long-term collaboration in improving supply chain performance. It also supports previous work which suggests that long-term collaboration does not enhance supply chain competitiveness. As presented in section 2, previous studies find that having a very close partnership with long-term collaboration is not more advantageous than having shorter partnerships with less close relationship (Parker and Hartley 1997). Leeuw and Fransoo (2009) also find that a close collaboration through long-term partnerships reflects an analogy that is "one size does not fit all". This means that a successful collaboration practice of a company does not always fit to other enterprises. Squire et al. (2009) also suggest that although duration of collaboration can improve manufacturer's responsiveness, the extremely long duration of collaboration turns the manufacturer to be dependent on the supplier. This situation may lead to an adverse impact on the overall demand fulfilment. In other words, from a market perspective, the benefit of establishing longer duration of collaboration does not improve the supply chains competitiveness over the long-term.

To obtain further explanations of this result, agents' movements during the simulation are observed in every time unit. It is found that both suppliers' and manufacturers' strategic movements are influenced by the model assumption, where the firms have no loyalty to their collaborating partner. As the supplier attracts a new manufacturer persistently, this assumption makes the supplier move consistently further from the manufacturer with whom it currently collaborates. This direction of the supplier's strategic movement enlarges the strategic gap between the manufacturer and the supplier which can lead to a higher risk for the supplier to lose the opportunity to reestablish collaboration with the previous manufacturer when the collaboration ends. Moreover, the manufacturer will select a new supplier if the previous supplier no longer meets the manufacturer's preference. This resulting behavior represents a situation where suppliers tend to be less cautious in making decision for their strategic move because they believe the longer term partnerships have secured their business for the long run in the future.

In contrast, the extreme short collaboration duration can prevent the supplier from moving further from the current manufacturer. This emergent behavioral pattern occurs when the supplier does not find another manufacturer to collaborate with while the previous manufacturer is still the closest agent to the supplier. In this situation, the supplier will move back to the previous strategic position to attract the manufacturer to rebuild the collaboration link. This pattern becomes prominent to demand fulfilment rate 
and survivability of the supply chains when the supplier can collaborate with more than one manufacturer. In this situation, the extremely short-term collaboration is found to provide better demand fulfilment and survivability of the supply chains than the longer duration of collaboration. In addition, although the suppliers are expected to have a longer "life" to exist in the system when they work with more than one manufacturer, this advantage is only significant when all supply chains in the market choose the strategy of a longer duration of collaboration.

On the other hand, in the model, a longer term of collaboration will limit the manufacturer's strategic movement as well as manufacturer's life when the supplier turns to be less efficient and/or less responsive during the collaboration period. This rule reflects a circumstance where long-term collaboration leads to the manufacturer becoming dependent on the supplier. When the manufacturer experiences losses during the interaction with the supplier, a longer duration of collaboration will jeopardize not only the manufacturer's survivability but also the supply chain's existence in the market. This is due to the decrease of the number of manufacturers, which eventually reduces the suppliers' market size.

To our knowledge, no study has attempted so far to compare the extent of demand fulfilment by varying the duration of collaboration. These findings provide a new perspective in understanding the significance of long-term effect of the longer duration of collaboration. Companies which currently consider establishing long-term partnerships with their suppliers may need to take into account their options. This findings confirm the strategic management view, where operational effectiveness does not lead to a sustainable competitive advantage (Porter 2006). Meanwhile, most SCM work observes collaboration from an operational perspective - without taking into account the emergent outcome of the firms' behavior on the market.

The limitations of this work should also be considered. In the model, all agents are assumed to be homogeneous, rationally bounded, and do not have learning ability to update their behavioral rules. Firms apply exactly the same length of collaboration during the simulation. Moreover, the model does not consider collaboration cost and operations measures that may provide a further insight on the supply chain performance. However, the simple model used in this research is found to be effective in exploring the problem from a market perspective.

\section{CONCLUSIONS}

This study examines the effect of duration of collaboration on supply chains by using an agent-based modeling $(\mathrm{ABM})$ approach which enables taking a market perspective in the analysis. The market perspective consists a novel approach in evaluating supply chains in the market. It provides further insights in SCM, particularly with regards to the impact of long-term collaboration on the market performance. The findings show that the benefits of adopting a longer duration of collaboration are limited to the individual supply chain perspective. The strategy does not lead to a long-term supply chain performance, in terms of market demand fulfilment and supply chain's ability to survive in the market. Thus, an essential insight gained from the application of market perspective in this study is that "what is good for a single company could be detrimental once all firms follow it ". This insight can encourage academics, business managers, and market regulators (policy makers) to review and improve their existing practice in supply chain strategy. The adoption of a longterm collaboration should be decided upon based on the supply and demand behavior, supplier's strategic position and movement, and competitors' behavior in the market.

While this study provides a novel approach and insights to understanding competition and collaborations in supply chains, the model offers potential extensions to generate further valuable comprehension and intuition in this topic. Future work could be performed by addressing the limitations of the model in assessing the effect of competition and collaboration in supply chains, such as allowing heterogeneous agents in the model. Incorporating more supply chain elements, such as number of partnerships and trust or loyalty between collaborating firms, could also enhance the interpretation of the current finding. Moreover, allowing agents to be heterogeneous by applying different duration of collaboration in each manufacturer agent could improve the insights gained through this paper.

\section{REFERENCES}

Anderson, E. and Jap, S.D., 2005. The dark side of close relationships. MIT Sloan Management Review, 46(3), pp. 7583.

Barratt, M., 2004. Understanding the meaning of collaboration in the supply chain. Supply Chain Management: An International Journal, 9(1), pp. 30-42.

Bensaou, M., 1999. Portfolios of buyer-supplier relationships. MIT Sloan Management Review, pp. 1-12.

Boddy, D. Cahill, C., Charles, M., Fraser-Kraus, H., and Macbeth, D., 1998. Success and failure in implementing supply chain partnering: an empirical study. European Journal of Purchasing and Supply Management, 4, pp. 143-151.

Burke, G.J., Carrillo, J.E. and Vakharia, A.J., 2007. Single versus multiple supplier sourcing strategies. European Journal of Operational Research, 182(1), pp. 95-112.

Cao, M. and Zhang, Q., 2011. Supply chain collaboration: Impact on collaborative advantage and firm performance. Journal of Operations Management, 29(3), pp. 163-180.

Cheng, R., 2011. A study on multi-agent based supply chain collaboration operation model facing time of delivery. Proceedings - 2011 8th IEEE International Conference on eBusiness Engineering, ICEBE 2011, pp. 102-104.

Chopra, S. and Meindl, P., 2007. Supply chain management: Strategy, planning, and operation, New Jersey: Pearson Education, Inc.

Christopher, M., 2000. The agile supply chain: Competing in volatile markets. Industrial Marketing Management, 1(29), pp. $37-44$

Collier, N., 2003. RePast: An Extensible Framework for Agent Simulation. The University of Chicago Social Science Research, 36, pp. 371-375.

Dapiran, P., 1992. Benetton - Global logistics in action. International Journal of Physical Distribution and Logistics Management, 22(6), pp. 7-11.

Dimitriou, S., 2010. The impact of prices on boundedly rational decision makers in supply chains. The University of Warwick.

Dyer, J.H. and Ouchi, W.G., 1993. Japanese-style partnerships: Giving companies a competitive edge. MIT Sloan Management Review, pp. 1-17. 
Grayson, K. and Ambler, T.I.M., 1999. The dark side of long-term relationships in marketing services. Journal of Marketing Research, XXXVI (1992), pp. 132-141.

Holweg, M. Disney, S., Holmström, J., and Småros, J., 2005. Supply chain collaboration: Making sense of the strategy Continuum. European Management Journal, 23(2), pp. 170 181.

Inkpen, A.C. and Beamish, P.W., 1997. Knowledge, bargaining power, and the instability of international joint ventures. Academy of Management Review, 22(1), pp. 177-202.

Jennings, N.R., Sycara, K. and Wooldridge, M., 1998. A Roadmap of Agent Research and Development. Autonomous Agents and Multi-Agent Systems, 1, pp. 7-38.

Jiao, J., You, X. and Kumar, A., 2006. An agent-based framework for collaborative negotiation in the global manufacturing supply chain network. Robotics and Computer-Integrated Manufacturing, 22(3), pp. 239-255.

Julka, N., Srinivasan, R. and Karimi, I., 2002. Agent-based supply chain management-1: framework. Computers and Chemical Engineering, 26(12), pp. 1755-1769.

Kraljic, P., 1983. Purchasing must become supply management. Harvard Business Review.

Lambert, D.M. and Cooper, M.C., 2000. Issues in supply chain management. Industrial Marketing Management, 29(1), pp. 65-83.

Leeuw, S. De and Fransoo, J., 2009. Drivers of close supply chain collaboration: one size fits all? International Journal of Operations and Production Management, 29(7), pp. 720 739.

Li, S. Ragu-Nathan, B., Ragu-Nathan, T. S., and Rao S.S., 2006. The impact of supply chain management practices on competitive advantage and organizational performance. Omega, 34(2), pp. 107-124.

Macal, C.M. and North, M.J., 2011. Introductory tutorial: Agentbased modeling and simulation. In M. F. S. Jain, R.R. Creasey, J. Himmelspach, K.P. White, ed. Proceedings of the 2011 Winter Simulation Conference. The Institute of Electrical and Electronics Engineers, Inc., pp. 1451-1464.

Matthyssens, P. and Van den Bulte, C., 1994. Getting closer and nicer: Partnerships in the supply chain. Long Range Planning, 27(1), pp. 72-83.

Onggo, B.S. and Karatas, M., 2015. Agent-based model of maritime search operations: A validation using test-driven simulation modelling. In L. Yilmaz et al., eds. Proceedings of the 2015 Winter Simulation Conference. IEEE, pp. 254-265.

Parker, D. and Hartley, K., 1997. The economics of partnership sourcing versus adversarial competition: a critique. European Journal of Purchasing and Supply Management, 3(2), pp. 115-125.

Porter, M.E., 1997. Competitive strategy. Measuring Business Excellence, 1(2), pp. 12-77.

Porter, M. E., 2006. What is strategy? In M. Mazzucato (Ed.), Strategy for business (10-30). Oxford: Sage Publications, Inc.
Prajogo, D. and Olhager, J., 2012. Supply chain integration and performance: The effects of long-term relationships, information technology and sharing, and logistics integration. International Journal of Production Economics, 135(1), pp. 514-522.

Ren, Z.J., Cohen, M.A., Ho, T.H., and Terwiesch, C., 2010. Information sharing in a long-term supply chain relationship: The role of customer review strategy. Operations Research, 58(1), pp. 81-93.

Robertson, D.A., 2003. The strategy hypercube: Exploring strategy space using agent-based models. Lecture Notes in Computer Science, 2927, pp. 182-192.

Robertson, D.A. and Caldart, A.A., 2009. The dynamics of strategy: Mastering strategic landscapes of the firm, New York, New York, USA: Oxford University Press.

Robinson, S., 2008. Conceptual modelling for simulation Part I: Definition and requirements. Journal of the Operational Research Society, 59(3), pp. 278-290.

Robinson, S., 2014. Simulation: The practice of model development and use Second, Palgrave Macmillan.

Squire, B. Cousins, P.D., Lawson, B., and Brown, S., 2009. The effect of supplier manufacturing capabilities on buyer responsiveness: The role of collaboration. International Journal of Operations and Production Management, 29(8), pp. 766-788.

Sun, J. and Debo, L., 2014. Sustaining long-term supply chain partnerships using price-only contracts. European Journal of Operational Research, 233(3), pp. 557-565.

Swaminathan, J.M., Smith, S.F. and Sadeh, N.M., 1998. Modeling supply chain dynamics: A multiagent approach. Decision Sciences, 29(3), pp. 607-632.

Tako, A.A. and Robinson, S., 2012. The application of discrete event simulation and system dynamics in the logistics and supply chain context. Decision Support Systems, 52(4), pp. 802-815.

Tyworth, J.E. and Ruiz-Torres, A., 2000. Transportation's role in the sole- versus dual-sourcing decision. International Journal of Physical Distribution \& Logistics Management, 30(2), pp. 128-144.

Wagner, S.M., 2011. Supplier development and the relationship life-cycle. International Journal of Production Economics, 129(2), pp. 277-283.

Wooldridge, M. and Jennings, N.R., 1995. Intelligent agents: theory and practice. The Knowledge Engineering Review, 10(2), pp. 115-152.

Xue, X. Li, X., Shen, Q., and Wang, Y., 2005. An agent-based framework for supply chain coordination in construction. Automation in Construction, 14(3), pp. 413-430.

Zhang, D.Z., Anosike, A.I., Lim, M.K., and Akanle, O.M., 2006. An agent-based approach for e-manufacturing and supply chain integration. Computers and Industrial Engineering, 51(2), pp. 343-360.

Zhu, X., 2008. Agent Based Modeling for Supply Chain Management: Examining the Impact of Information Sharing. Kent State University.

Niniet Indah Arvitrida is a Lecturer in Department of Industrial Engineering, Sepuluh Nopember Institute of Technology (ITS), Indonesia. Before taking on a PhD in Simulation at Loughborough University, UK, she was working in academia and being a professional trainer in production management, material management, and logistics and supply chain management for corporates and small and medium enterprises in Indonesia. She also has a Master of Engineering in Supply Chain Management and Bachelor of Engineering in Industrial Engineering, both obtained with honors (cum laude) in ITS, Indonesia.

Antuela A. Tako is a Senior Lecturer in Operational Research at the School of Business and Economics, Loughborough University, UK. She holds a PhD in Simulation and an MSc in Management Science and Operational Research from the University of Warwick, UK. Antuela is a member of the EPSRC Peer Review College, UK an Associate Fellow of the UK's Operational Research Society (AFORS). Her research interests include the comparison of alternative simulation approaches, 
facilitated and participative simulation modeling and conceptual modeling. Antuela is a co-founder of the PartiSim framework (www.partisim.org.uk). Her email address is: a.takou@lboro.ac.uk

Duncan Robertson is a Lecturer in Management Science at the School of Business and Economics, Loughborough University, UK. Before undertaking his DPhil at Saïd Business School at the University of Oxford, UK, he qualified as a Chartered Accountant with KPMG working in the City of London, Australia, and New Zealand, where he specialized in advisory and corporate finance roles within the banking sector. He originally trained as a physicist at Imperial College London. Home page: http://www.lboro.ac.uk/ departments/sbe/staff/profiles/robertsonduncan/ robertson-duncan.html.

Stewart Robinson is Professor of Management Science and Dean at the School of Business and Economics, Loughborough University, UK. He holds an honors degree in Management Science (Operational Research) (1985) and a PhD in Management Science (1998), both from Lancaster University. His research interests are in the practices and use of simulation models where he focuses on conceptual modelling, model validation, output analysis and alternative simulation methods (discrete-event, system dynamics and agent based). Home page: www.stewartrobinson.co.uk. 